\title{
Precision Open-Ended Coaxial Probes for In Vivo and Ex Vivo Dielectric Spectroscopy of Biological Tissues at Microwave Frequencies
}

\author{
Dijana Popovic, Student Member, IEEE, Leah McCartney, Student Member, IEEE, \\ Cynthia Beasley, Student Member, IEEE, Mariya Lazebnik, Student Member, IEEE, \\ Michal Okoniewski, Senior Member, IEEE, Susan C. Hagness, Senior Member, IEEE, and \\ John H. Booske, Senior Member, IEEE
}

\begin{abstract}
Hermetic stainless-steel open-ended coaxial probes have been designed for precision dielectric spectroscopy of biological tissue, such as breast tissue, over the $0.5-20-\mathrm{GHz}$ frequency range. Robust data-processing techniques have also been developed for extracting the unknown permittivity of the tissue under test from the reflection coefficient measured with the precision probe and a vector network analyzer. The first technique, referred to as a reflection-coefficient deembedding method, converts the reflection coefficient measured at the probe's calibration plane to the desired aperture-plane reflection coefficient. The second technique uses a rational function model to solve the inverse problem, i.e., to convert the aperture-plane reflection coefficient to the tissue permittivity. The results of the characterization and validation studies demonstrate that these precision probes, used with the prescribed measurement protocols and data-processing techniques, provide highly accurate and reliable in vivo and $e x$ vivo biological tissue measurements, including breast tissue spectroscopy.
\end{abstract}

Index Terms-Breast tissue, dielectric properties of biological tissue, microwave measurements, open-ended coaxial probe, rational function model (RFM), vector network analyzer (VNA).

\section{INTRODUCTION}

A LARGE-SCALE collaborative study between the University of Calgary (UC), Calgary, AB, Canada, and the University of Wisconsin-Madison (UW) [1] aims to build an extensive database of the dielectric properties of various normal and diseased breast tissue at microwave frequencies. This data may facilitate further developments of new microwave diagnostic methods for breast cancer detection [2]-[12] and microwave hyperthermia treatment of breast cancer [13], [14]. The data in the $0.5-20-\mathrm{GHz}$ frequency range are collected ex vivo and in vivo using open-ended coaxial probes as sensors.

Open-ended coaxial probes have been used extensively in dielectric spectroscopy of biological tissues due to their

Manuscript received August 6, 2004. This work was supported by the National Institutes of Health under Grant R01 CA 087007 awarded by the National Cancer Institute, by the National Science Foundation under a Graduate Research Fellowship, by the Natural Sciences and Engineering Research Council of Canada, and by iCORE Alberta.

D. Popovic, L. McCartney, and M. Okoniewski are with the Department of Electrical and Computer Engineering, University of Calgary, Calgary, AB, Canada T2N 1N4 (e-mail: michal@enel.ucalgary.ca).

C. Beasley, M. Lazebnik, S. C. Hagness, and J. H. Booske are with the Department of Electrical and Computer Engineering, University of Wisconsin-Madison, Madison, WI 53706 USA (e-mail: hagness@engr.wisc.edu).

Digital Object Identifier 10.1109/TMTT.2005.847111 simplicity, broad-band response, and capacity for noninvasive measurements [15], [16]. The probe is typically used in conjunction with a vector network analyzer (VNA) to record the frequency-dependent complex reflection coefficient. The VNA-probe system is first calibrated so that the reflection-coefficient measurements are referenced to the probe aperture plane. This is usually done in one of two ways, which are: 1) using reference liquids for direct calibration at the open end of the probe or 2) using mechanical or electronic calibration standards at the connector plane combined with a model of the probe that translates the reference plane to the aperture. The permittivity is then calculated from the reflection coefficient at the probe aperture using standard inverse techniques [17].

The accuracy and reliability of the measured data are closely related to the precision of the physical characteristics of the probe's aperture because it is the only part of the system in direct contact with the tissue. Teflon-filled copper probes, conventionally used for broad-band reflection coefficient measurements, can yield inaccurate or unreliable measurements. The aperture may physically deteriorate during the handling of the probe [18], resulting in worst case measurement errors in the reflection coefficient as large as $30 \%$ at $20 \mathrm{GHz}$. In a hospital environment, the aperture is affected by variations between the air and sample temperatures, chemical reactions between the fluid in the tissue samples and copper, and leakage of these fluids into the aperture. Furthermore, Teflon-filled copper probes neither meet bio-compatibility requirements, nor can they endure the harsh sterilization process required for in vivo measurements. For all of these reasons, we concluded that a more robust probe was needed to ensure the integrity of our breast tissue dielectric spectroscopy study.

A hermetic stainless-steel borosilicate-glass-filled coaxial probe was designed to mitigate the problems inherent with copper-Teflon probes [19]. Several of these "precision" probes were commercially manufactured following our specifications. ${ }^{1}$ The materials used to build the precision probe are chemically inert and thermally matched. Furthermore, the probe was hermetically sealed at the aperture, making it robust in a hospital environment. Possible measurement scenarios include ex vivo measurements of freshly excised tissues in pathology, invasive in vivo measurements of tissues during surgical procedures, as

\footnotetext{
${ }^{1}$ The probes were manufactured by a division of the Kaman Aerospace Corporation, which became Meggitt Safety Systems Inc., Simi Valley, CA. [Online] Available: http://www.meggittsafety.com/
} 


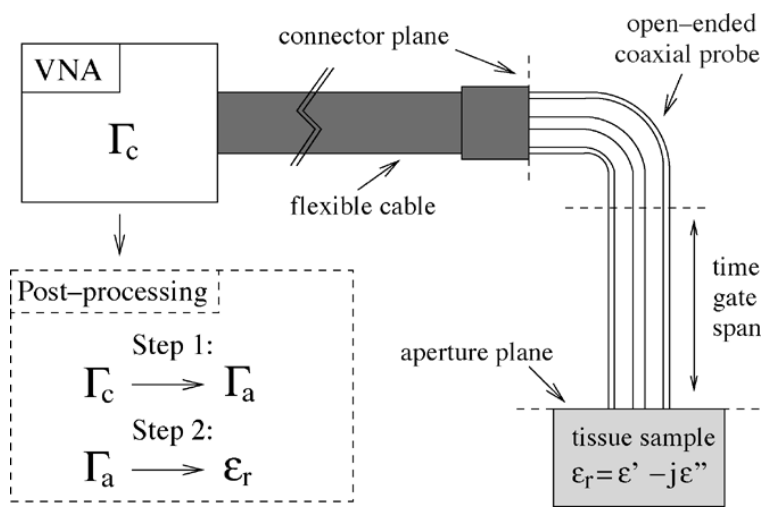

Fig. 1. Overview of the dielectric characterization protocol using a precision open-ended coaxial probe. Reflection coefficient data is acquired by the VNA, referenced to the connector/calibration plane. Tissue dielectric properties are calculated using two data-processing steps.

well as noninvasive in vivo measurements of exposed tissues such as the skin. The probe is flange free to ensure good contact with tissue samples across the whole aperture. The outer conductor walls near the probe aperture are thickened for increased accuracy [20]. Based on data from a previous study [21], the probe aperture diameter is set to $3.0 \mathrm{~mm}$ to provide optimum sensitivity in both the high- and low-permittivity tissue measurements. The minimum length of the probe is dictated by the time resolution of the VNA [22]. The length of the precision probe was set to $10 \mathrm{~cm}$ to clearly differentiate between the reflection at the aperture and the reflection at the connector in the time-domain trace.

Rigorous measurement protocols were developed for the precision probe. As shown in Fig. 1, the VNA is calibrated at the connector plane using calibration standards and time gating [22], [23]. This procedure avoids the uncertainties associated with reference-liquid calibration. The complex reflection coefficient data $\Gamma_{c}$, referenced to the connector plane, are recorded and post-processed in two steps. In the first step, a deembedding model is used to compensate for the propagation effects of the precision probe and translate the measurement reference plane to the probe aperture and derivce the deembedded reflection coefficient $\Gamma_{a}$. In the second step, a rational function model (RFM) [23], [24] is applied to the deembedded reflection coefficient to calculate the permittivity and conductivity of the breast tissue specimen across the frequency range of interest. This measurement system and protocol are applicable to characterizing other biological tissues, as well as measurements of other soft or liquid materials that require a probe to perform robustly in a challenging environment.

The remainder of this paper is organized as follows. Section II describes the details of the probe construction and derivation of the reflection-coefficient deembedding model. This derivation relies upon careful finite-difference time-domain (FDTD) simulations [25] of an idealized precision probe immersed in reference liquids, as well as experimental measurements of those same reference liquids. The liquids selected were deionized water, methanol, and ethanol since their dielectric properties span the expected range of breast tissue permittivity. Section III describes the derivation of the RFM for the precision probes, which relies upon FDTD simulations of the idealized

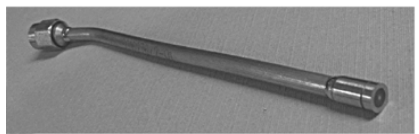

(a)

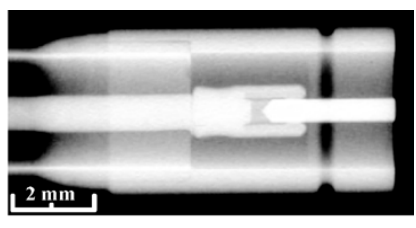

(b)
Fig. 2. (a) Precision open-ended coaxial probe. The probe is $10-\mathrm{cm}$ long and $4 \mathrm{~mm}$ in diameter at the aperture. (b) X-ray image revealing the internal configuration near the aperture end of the probe.

probe immersed in lossless nondispersive materials. Section IV presents results that validate the deembedding model and the RFM separately. Also presented are the results of measurements with the reference liquids and a test liquid-a mixture of ethanol and methanol-that verify the measurement protocol in which the deembedding model and RFM are used together.

\section{DeEmbedding Model of The Probe}

Fig. 2 shows a photograph and an X-ray image of a precision probe constructed with several sections of high-quality $50-\Omega$ coaxial lines. The first section following the connector is a 9.5-mm-long coaxial line filled with low-loss porous silica $\left(\epsilon_{r}=1.72\right)$. The last section (at the probe aperture) is a 2.3-mmlong coaxial line filled with a borosilicate glass $\left(\epsilon_{r}=3.75\right)$. At the aperture plane, the radii of the inner and outer conductors were specified to be 0.3 and $1.5 \mathrm{~mm}$, respectively, while the probe's outer radius is $2.1 \mathrm{~mm}$. The borosilicate glass is hermetically sealed to the stainless-steel conductors. The thermal expansion coefficients of the borosilicate and stainless steel are low and matched (3.0 to $3.4 \times 10^{-6}$ and $5.0 \times 10^{-6}$, respectively, ${ }^{2}$ compared to $17.7 \times 10^{-6}$ and $100 \times 10^{-6}$ for copper and Teflon [26]). A 2.9-mm air-filled line is used as the transition between the two glass lines. Furthermore, the inner conductor of the probe is protruding slightly, a technique used to achieve more accurate low-permittivity measurements [27].

The multisection construction greatly reduces the cost, but complicates the calibration procedures, as it gives rise to parasitic capacitances and inductances in the transition regions. The design of the air transition is such that the two parasitic effects should cancel out; however, due to variations in the manufacturing process (particularly welding), this cancellation is not perfect, especially when the large bandwidth required by our spectroscopy application is considered. Time gating can be used to remove unwanted reflections at the connector. However, as shown in Fig. 3, the small reflections (on the order from -40 to $-30 \mathrm{~dB}$ ) arising from the discontinuities at the airline section are temporally too close to the desired response peak to be gated out. Moreover, the precise dimensions of the air-filled section can differ between probes due to the welding process.

A reflection-coefficient deembedding model was developed to compensate for the propagation characteristics of the multisection probe. This model permits the extraction of the desired reflection coefficient at the aperture plane from the one measured at the calibration plane. The required accuracy of the

\footnotetext{
${ }^{2}$ Internet resources. Accessed Nov. 2001. [Online]. Available: http://www.glassalchemyarts.com/glossary.html, www.gocarlson.com/AlloyPDF/Nitronic50.pdf
} 


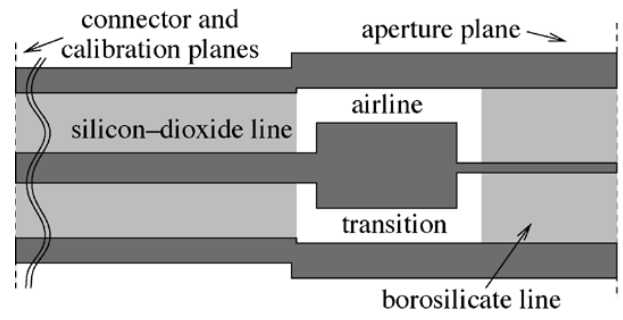

(a)

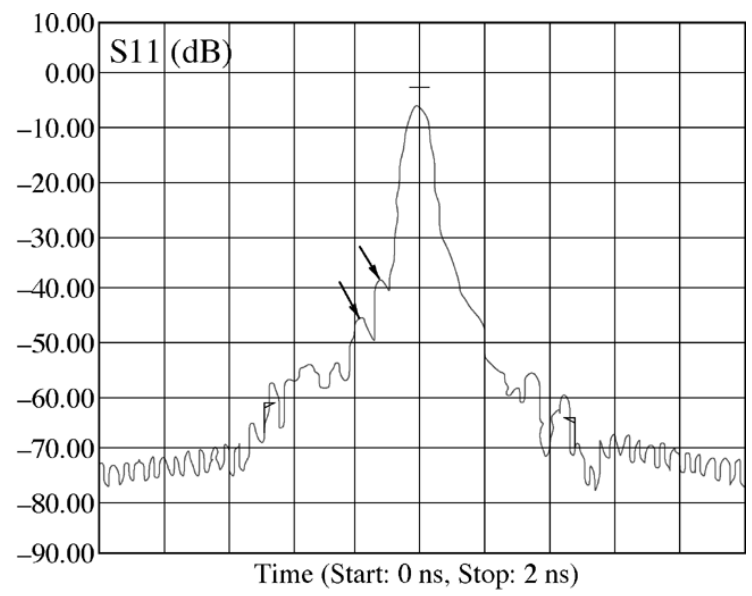

(b)

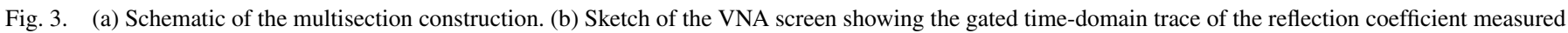

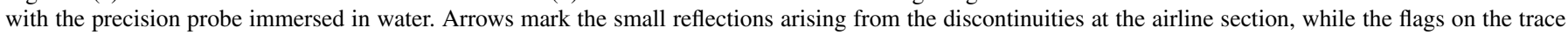
mark the center and the span of the time gate used to remove the connector reflections.

deembedding model was determined from a previous sensing volume study performed by our group [21]. In that study, we found that in order to limit the level of error in the real $\left(\epsilon^{\prime}\right)$ and imaginary $\left(\epsilon^{\prime \prime}\right)$ parts of the measured complex permittivity to $10 \%$, the errors in the magnitude and phase of the aperture-plane reflection coefficient should be less than $\sim 1 \%$ and $\sim 1^{\circ}$, respectively. (The specific reflection coefficient error thresholds vary slightly depending on the permittivity of the sample under test.) Therefore, we chose error thresholds of $\sim 1 \%$ (magnitude) and $\sim 1^{\circ}$ (phase) as the accuracy target for the deembedded reflection coefficient. A unique deembedding model was determined for each of the probes to achieve the same accuracy levels in spite of dimensional variances.

In previous research, a lumped-element approach was adopted for the deembedding model [19], [28]. That approach used lumped elements and transmission lines to capture the heterogenenity of the probe configuration. The derivation of the lumped-element model was limited by our ability to accurately measure the probe's internal dimensions, specifically the dimensions of the airline transition, from the X-ray images and the assumption of perfect axial symmetry of the welding process. Multiple frequency ranges were required to obtain the best fit element values.

Here, we have adopted an alternative approach for the deembedding model. This approach treats the probe as a two-port microwave network [29], wherein $S$-parameters are used to relate the reflection coefficient at the connector/calibration plane $\Gamma_{c}$ to the reflection coefficient at the aperture plane $\Gamma_{a}$ as follows:

$$
\Gamma_{c}=S_{11}+\frac{S_{12} S_{21} \Gamma_{a}}{1-S_{22} \Gamma_{a}} .
$$

This equation was derived using the well-known signal flow graph shown in Fig. 4 (see e.g., Pozar, Microwave Engineering).

To determine the unknown $S$-parameters in (1), we used measured data for $\Gamma_{c}$ and simulated data for $\Gamma_{a}$. The calibrationplane reflection coefficients were measured with each probe in air and the three reference liquids (deionized water, methanol, and ethanol). Since it is difficult to position the probe exactly on

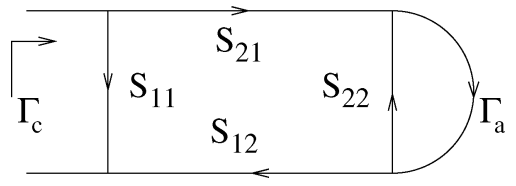

Fig. 4. Signal flow graph for the probe modeled as a two-port microwave network.

the surface of the liquid samples, we decided to fully immerse the tip of the probes into the liquids, thereby removing all uncertainties involving the positioning of the probes. This probe configuration differed slightly from the configuration encountered in practice where the probe tip is pressed against the surface of the semisolid tissue specimen, as illustrated in Fig. 1. However, a previous study published by our group demonstrated that this slight difference in configuration had no significant effect on the relevant sensing characteristics of the probe [21]. The reflection-coefficient measurements were taken on three different days and recorded together with the temperature of the immersion medium. Thus, for each probe, we generated three sets of data containing four measurements each.

The aperture-plane reflection coefficients were computed using two-dimensional (2-D) body-of-revolution (BOR) FDTD simulations of an idealized probe immersed in each reference liquid in a manner similar to [20]. As shown in Fig. 5, the model of the idealized probe assumed that the coaxial line was homogeneous; i.e., the radial dimensions were uniform along the entire length of the probe and the probe was filled entirely with borosilicate glass. The radial dimensions of the idealized probe were chosen to represent those of the aperture end of the manufactured probe, as estimated from the X-ray images. The inner- and outer-conductor radii for the primary UC probe were determined to be $a=0.291 \mathrm{~mm}$ and $b_{2}=1.475 \mathrm{~mm}$, respectively. For the primary UW probe, we measured the following dimensions: $a=0.293 \mathrm{~mm}$ and $b_{2}=1.478 \mathrm{~mm}$. An extremely fine grid resolution, $12.5 \times 12.5 \mu \mathrm{m}$, was required to accurately model the fringing fields at the flange-free probe aperture. This grid resolution was determined through a series of reflection-coefficient convergence tests with varying grid sizes. 


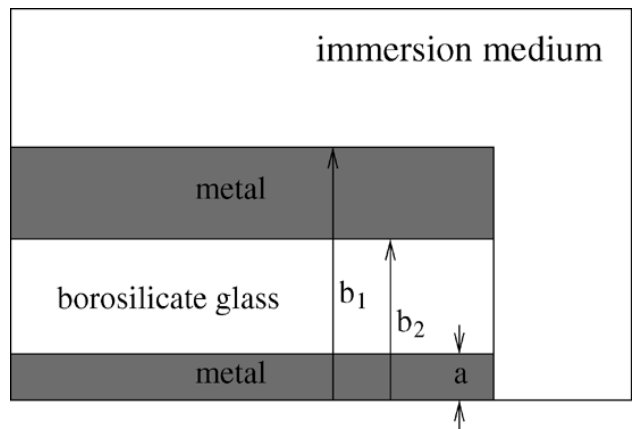

Fig. 5. 2-D BOR FDTD model of the idealized probe used in the derivations of the deembedding model and RFM. The radii of the inner and outer conductors are denoted as $a$ and $b_{2}$, respectively. The outer diameter of the probe is denoted as $b_{1}$.

Within the limits of this extremely fine grid discretization, the two primary probes used in this study were modeled with the same radial dimensions ( $a=0.2875 \mathrm{~mm}$ and $\left.b_{2}=1.475 \mathrm{~mm}\right)$, as these dimensions represented the closest multiple of $12.5 \mu \mathrm{m}$ to the actual dimensions. The known dielectric properties of the reference liquids were incorporated into the FDTD simulations using Debye dispersion parameters taken from [30]. The Debye parameters were interpolated to fit the experimentally measured temperature conditions by assuming a locally linear temperature dependence.

For each probe, a MAPLE LeastSquares function with recursion was used to determine the $S$-parameters that optimally fit one of the three measurement data sets $\left(\Gamma_{c}\right)$ to the corresponding simulation data set $\left(\Gamma_{a}\right)$ for the frequency range of interest. The frequency range used in this optimization procedure was $0.1-20$ $\mathrm{GHz}$. This range is larger than the actual frequency range of interest $(0.5-20 \mathrm{GHz})$. The low-frequency margin was introduced to ensure smooth results down to $0.5 \mathrm{GHz}$. Since the transmission $S$-parameters appear as a product in (1), the least squares fitting process required the determination of a total of only three independent parameters for each data set. This fitting process was repeated for the remaining two data sets, and the three sets of $S$-parameters were averaged. At this point, the derivation of the probe-specific deembedding model was completed.

Finally, to use the deembedding model as a data processing tool, we inverted (1) as follows:

$$
\Gamma_{a}=\frac{1}{S_{22}+\frac{S_{12} S_{21}}{\left(\Gamma_{c}-S_{11}\right)}} .
$$

Equipped with the optimized set of frequency-dependent $S$-parameters, this model deembeds an unknown aperture-plane reflection coefficient $\Gamma_{a}$ from the reflection coefficient measured at the connector/calibration plane $\left(\Gamma_{c}\right) . \Gamma_{a}$ is converted to an aperture admittance and used as input data in the next data processing step, as discussed in Section III.

\section{RFM OF THE PROBE}

The RFM developed by Stuchly et al. [23] and Anderson et al. [24] is a closed-form analytic model of the inverse problem used to determine the permittivity of a dielectric medium from the measured reflection coefficient of an open-ended coaxial probe. The model was derived assuming an idealized configuration of a
$50-\Omega$ semirigid Teflon-filled coaxial line with an infinite flange radiating into an infinite homogeneous dielectric medium. It was determined to be valid in the $1-20-\mathrm{GHz}$ frequency range for $1 \leq \epsilon_{r}^{\prime} \leq 80$ and $0 \leq \epsilon_{r}^{\prime \prime} \leq 80$, where $\epsilon_{r}=\epsilon_{r}^{\prime}-j \epsilon_{r}^{\prime \prime}$ is the complex dielectric constant of the medium under test.

Several features of our probes rendered the previous RFM unsuitable for our application to the dielectric spectroscopy of breast tissue. Absence of the flange on the precision probes ensures better contact with the heterogeneous breast tissue specimens across the aperture, but also gives rise to fringing effects that are not present in the models derived by Stuchly et al.and Anderson et al. In addition, the precision probes are filled with borosilicate glass at the aperture and the frequency range of interest extends down to $500 \mathrm{MHz}$. Thus, we adapted the procedure proposed in [24] and derived an RFM model specifically for the precision probes.

The relationship between $\varepsilon_{r}$ and the aperture admittance $Y^{\text {Model }}$ is given by the following RFM:

$$
Y^{\mathrm{Model}}\left(s, \epsilon_{r}\right) \cong \frac{\sum_{n=1}^{N} \sum_{p=1}^{P} \alpha_{n p}\left(\sqrt{\epsilon_{r}}\right)^{p}(s a)^{n}}{1+\sum_{m=1}^{M} \sum_{q=0}^{Q} \beta_{m q}\left(\sqrt{\epsilon_{r}}\right)^{q}(s a)^{m}}
$$

where $s$ is the complex frequency, $a$ is the radius of the inner conductor of the coaxial line, and $\alpha_{n p}$ and $\beta_{m q}$ are the model coefficients [24].

To determine the unknown model coefficients in (3), we performed a nonlinear least squares fit to minimize the difference between the admittances predicted by the RFM (3) for a set of known permittivities and the admittances computed by 2-D BOR FDTD simulations of the probe immersed in media with the same permittivities. The idealized homogeneous coaxial line model described in Section II and illustrated in Fig. 5 was assumed here as well. However, since our goal was to create a general RFM that was insensitive to minor dimensional variances introduced in the construction of the probes, we simulated a probe with generic radial dimensions corresponding to the design specifications (i.e., $a=0.3 \mathrm{~mm}$ and $b_{2}=1.5 \mathrm{~mm}$, respectively). The idealized probe was assumed to be immersed in a nondispersive lossless dielectric medium with $1 \leq \epsilon_{r}^{\prime} \leq 80$ and $\epsilon_{r}^{\prime \prime}=0$. Each simulation produced a broad-band reflection coefficient computed at the aperture plane.

A MATLAB function that implements the Levenberg-Marquardt algorithm was used to perform the fit using a total of 4000 data points representing 100 discrete frequencies in the range $0.1 \leq f \leq 20 \mathrm{GHz}$ and 40 dielectric constants in the range $1 \leq \epsilon_{r}^{\prime} \leq 80$. For reasons similar to those discussed in Section II, the frequency range used here in the optimization of the RFM was larger than the actual range of interest. The following merit function was used for the nonlinear least squares fit [24]:

$$
\chi^{2}=\sum_{i=1}^{4000}\left|\frac{Y_{i}^{\mathrm{FDTD}}-Y_{i}^{\mathrm{Model}}}{0.01 \cdot Y_{i}^{\mathrm{FDTD}}}\right| .
$$

$Y_{i}^{\text {FDTD }}$ and $Y_{i}^{\text {Model }}$ represent the admittance computed via FDTD and (3), respectively, for each $\left(s_{i}, \epsilon_{r_{i}}^{\prime}\right)$.

After the coefficients were determined, the solution of the RFM inverse problem proposed in [24] was used as the 


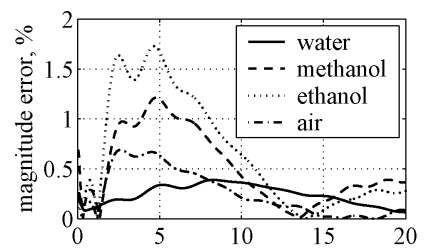

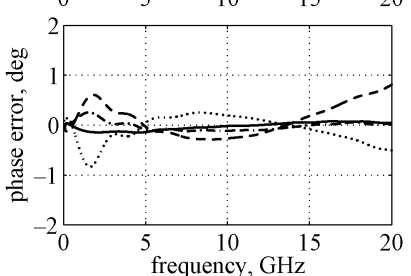

(a)
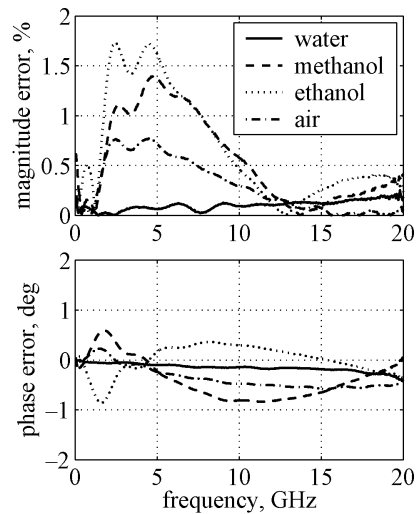

(b)
Fig. 6. Error between the experimental and theoretical aperture-plane reflection coefficients for air, water, methanol, and ethanol. The experimental data was generated from the reflection coefficient measured at the connector/calibration plane using the $S$-parameter-based deembedding model for: (a) the primary UC probe and (b) the primary UW probe.

data-processing tool to obtain the complex permittivity of an unknown material from an experimental determination of the probe's aperture admittance.

\section{RESULTS}

Here, we report the derived model parameters and the results of studies that were conducted to validate the use of the models in processing the reflection-coefficient data. We conducted performance evaluations of each of the two data-processing steps individually. We then evaluated the performance of the entire measurement protocol for determining the complex permittivity of reference and test liquids.

\section{A. Validation of the Deembedding Model}

The $S$-parameter optimization procedure described in Section II was used to derive a deembedding model separately for each probe. The magnitude of the resulting $S$-parameters ranged from 0 to 1 , as they should. While the optimized transmission parameter $\left(S_{12} S_{21}\right)$ for the primary UC probe was almost identical to that for the primary UW probe, the $S_{11^{-}}$and $S_{22}$-parameters between the two probes differed significantly. These observed differences confirmed the necessity of probe-specific deembedding models.

To validate the deembedding model for each probe, we compared the deembedded experimental aperture-plane reflection coefficient with the corresponding theoretical aperture-plane reflection coefficient for each reference liquid, as well as air. The deembedded experimental values of $\Gamma_{a}$ were obtained by applying (2) with the optimized probe-specific $S$-parameters to experimental values of $\Gamma_{c}$ measured with a specific probe. The theoretical values of $\Gamma_{a}$ were computed using FDTD simulations of the idealized probe. The error between the experimental and theoretical aperture-plane reflection coefficients is plotted in Fig. 6 for the primary UC probe and primary UW probe. The errors between the deembedded and theoretical reflection coefficients are less than $1.7 \%$ and $1^{\circ}$ in magnitude and phase, respectively, across the entire frequency range of interest $(0.5-20 \mathrm{GHz})$. The relative errors in the magnitude of the reflection coefficient were highest for ethanol. Further discussion of the impact of ethanol on the accuracy of the deembedding model is provided in Section $\mathrm{V}$.

The variability in published dielectric properties for reference liquids introduces some inevitable uncertainty into the derivation of the deembedding model. To verify the accuracy of the values used in the derivation of our deembedding model, we performed a set of precise measurements aimed at establishing the dielectric properties of the specific reference liquids used in our study. Reflection experiments in rectangular waveguides were selected because of the availability of analytical formulas relating dielectric properties and reflection coefficients, and the attainability of a very precise experimental setup. A one-port test network was developed using $X$ - and $K$-band waveguides. Two waveguides were used in each experiment, one for excitation and one as a holder for the reference liquid. A delrin seal was precision machined into the end of the excitation waveguide at the calibration plane. The end of each waveguide and seal was polished, tightly fitted, and aligned to inhibit liquid leakage and to prevent higher order modes from being excited inside the liquid-filled waveguide section. Best fit Cole-Cole parameters were calculated from the measured reflection coefficient data using nonlinear least squares fitting in MATLAB. For all three reference liquids, the Cole-Cole parameters determined from these optimizations produced complex permittivity results that were within $2 \%$ and $0.5^{\circ}$ in magnitude and phase of the respective temperature-interpolated permittivity used in the FDTD simulations. These results further confirmed the validity of the dielectric properties assumed in the derivation of the deembedding model.

The measurement protocol presented here provides sufficient accuracy for our spectroscopy application of interest. Nevertheless, it was of general interest to explore the possibility of making further improvements to the accuracy based on a more realistic model of the probe aperture. As mentioned previously, the inner conductor of the probe protrudes slightly at the aperture end (in the range of 10-20 $\mu \mathrm{m}$ ); however, this feature was not included in the FDTD simulations conducted for the deembedding model. In a follow-up study, we repeated those simulations with a modified FDTD model of the probe that included the protrusion. The modeling of the protrusion was made possible because of the extremely fine grid resolution used in the FDTD mesh. We found that the use of these more realistic FDTD simulations improved the performance of the deembedding model, particularly for ethanol (in fact, the error in the magnitude of the deembedded reflection coefficient was below $1 \%$ for all three reference liquids). Other small imperfections and manufacturing artifacts at the aperture could also be modeled using either the BOR FDTD grid or a full three-dimensional FDTD grid in cylindrical coordinates. However, the issue of where to set the reference plane for the deembedded reflection coefficient and RFM admittance model arises and a more sophisticated RFM model would be required.

\section{B. Validation of the RFM Model}

The smallest error for the RFM nonlinear least squares fit merit function was obtained for $N=M=6$ and $P=Q=8$. This is a larger number of RFM coefficients than that required 
TABLE I

BEST FIT $\alpha_{n p}$ COEFFICIENTS FOR $Y^{\mathrm{Model}}$

\begin{tabular}{c|c|c|c|c|c|c}
\hline $\mathrm{n}$ & 1 & 2 & 3 & 4 & 5 & 6 \\
$\mathrm{p}$ & & & & & & \\
\hline 1 & $-3.7421052 \mathrm{e}-01$ & $-4.2039090 \mathrm{e}-01$ & $-1.3373811 \mathrm{e}+00$ & $-1.7862154 \mathrm{e}+02$ & $1.0000000 \mathrm{e}+02$ & $1.0000000 \mathrm{e}+03$ \\
\hline 2 & $3.4704363 \mathrm{e}+00$ & $2.5791317 \mathrm{e}+00$ & $-1.3626541 \mathrm{e}+02$ & $-1.2385077 \mathrm{e}+02$ & $1.0000000 \mathrm{e}+04$ & $1.0000000 \mathrm{e}+04$ \\
\hline 3 & $-8.3855134 \mathrm{e}-01$ & $2.5168409 \mathrm{e}+01$ & $2.8973031 \mathrm{e}+02$ & $4.6871376 \mathrm{e}+02$ & $-1.0000000 \mathrm{e}+03$ & $1.0000000 \mathrm{e}+05$ \\
\hline 4 & $2.8353757 \mathrm{e}-01$ & $-3.4096359 \mathrm{e}+00$ & $-1.2845293 \mathrm{e}+02$ & $-1.4986977 \mathrm{e}+02$ & $9.9999999 \mathrm{e}+02$ & $-1.0000000 \mathrm{e}+04$ \\
\hline 5 & $-6.8318248 \mathrm{e}-02$ & $8.3756735 \mathrm{e}-01$ & $4.9067925 \mathrm{e}+01$ & $1.0245764 \mathrm{e}+01$ & $-1.0000005 \mathrm{e}+02$ & $1.0000000 \mathrm{e}+04$ \\
\hline 6 & $1.0738472 \mathrm{e}-02$ & $-3.3884325 \mathrm{e}-01$ & $-6.3007222 \mathrm{e}+00$ & $2.9793708 \mathrm{e}+01$ & $9.9999692 \mathrm{e}+01$ & $-9.9999999 \mathrm{e}+02$ \\
\hline 7 & $-9.2798364 \mathrm{e}-04$ & $2.2368933 \mathrm{e}-01$ & $1.9317092 \mathrm{e}-01$ & $-3.9523552 \mathrm{e}+00$ & $9.9983789 \mathrm{e}+00$ & $1.0000002 \mathrm{e}+02$ \\
\hline 8 & $3.2613792 \mathrm{e}-05$ & $-4.9217112 \mathrm{e}-03$ & $6.0334814 \mathrm{e}-01$ & $3.9807516 \mathrm{e}-02$ & $9.9121765 \mathrm{e}-01$ & $-9.9998826 \mathrm{e}+00$ \\
\hline
\end{tabular}

TABLE II

BEST FIT $\beta_{m q}$ COEFFICIENTS FOR $Y^{\text {Model }}$

\begin{tabular}{c|c|c|c|c|c|c}
\hline $\begin{array}{c}\mathrm{m} \\
\mathrm{q}\end{array}$ & 1 & 2 & 3 & 4 & 5 & 6 \\
\hline 0 & $1.2975091 \mathrm{e}+00$ & $-6.2123288 \mathrm{e}+01$ & $-2.4213389 \mathrm{e}+02$ & $-1.3012969 \mathrm{e}+03$ & $1.0000000 \mathrm{e}+04$ & $-1.0000000 \mathrm{e}+05$ \\
\hline 1 & $9.1790171 \mathrm{e}+00$ & $1.2909714 \mathrm{e}+02$ & $3.6142730 \mathrm{e}+02$ & $2.9512315 \mathrm{e}+03$ & $1.0000000 \mathrm{e}+04$ & $1.0000000 \mathrm{e}+06$ \\
\hline 2 & $4.8707509 \mathrm{e}-01$ & $-4.0602949 \mathrm{e}+01$ & $-9.5503416 \mathrm{e}+01$ & $-3.0523927 \mathrm{e}+03$ & $1.0000000 \mathrm{e}+04$ & $-1.0000000 \mathrm{e}+06$ \\
\hline 3 & $-4.5108600 \mathrm{e}-01$ & $2.1265024 \mathrm{e}+01$ & $6.8950920 \mathrm{e}+01$ & $2.1045700 \mathrm{e}+03$ & $-1.0000000 \mathrm{e}+04$ & $1.0000000 \mathrm{e}+06$ \\
\hline 4 & $-1.0598753 \mathrm{e}-01$ & $-2.5827272 \mathrm{e}+00$ & $8.1553263 \mathrm{e}+00$ & $-8.0781555 \mathrm{e}+02$ & $9.9999999 \mathrm{e}+02$ & $-1.0000000 \mathrm{e}+05$ \\
\hline 5 & $1.8503382 \mathrm{e}-01$ & $2.4954033 \mathrm{e}-01$ & $1.6618847 \mathrm{e}+00$ & $2.0962207 \mathrm{e}+02$ & $-1.0000000 \mathrm{e}+03$ & $1.0000000 \mathrm{e}+04$ \\
\hline 6 & $-2.8090331 \mathrm{e}-02$ & $1.6276127 \mathrm{e}-01$ & $-2.1284678 \mathrm{e}-01$ & $-2.5173013 \mathrm{e}+01$ & $1.0000024 \mathrm{e}+02$ & $-1.0000000 \mathrm{e}+03$ \\
\hline 7 & $3.2193221 \mathrm{e}-03$ & $2.7711524 \mathrm{e}-02$ & $1.2460695 \mathrm{e}+00$ & $1.4939035 \mathrm{e}+00$ & $-9.9980930 \mathrm{e}+00$ & $9.9999960 \mathrm{e}+01$ \\
\hline 8 & $-1.3862214 \mathrm{e}-04$ & $-1.6074907 \mathrm{e}-03$ & $3.8400381 \mathrm{e}-03$ & $-1.5851766 \mathrm{e}-01$ & $1.1404195 \mathrm{e}-01$ & $-1.0002079 \mathrm{e}+00$ \\
\hline
\end{tabular}

for the flanged copper-Teflon probe in [24], where $N=M=4$ and $P=Q=6$. We believe that the more complex fringing field patterns at the aperture of the flange-free precision probe dictated the larger number of coefficients. The RFM coefficients for the precision probe are summarized in Tables I and II.

We computed the magnitude of the relative error between the aperture admittance predicted by the RFM and the theoretical aperture admittance obtained from FDTD simulations as follows:

$$
\Delta Y=\frac{\left|Y^{\mathrm{Model}}-Y^{\mathrm{FDTD}}\right|}{\left|Y^{\mathrm{FDTD}}\right|} .
$$

This error is plotted in Fig. 7 versus frequency and dielectric constant. The relative error is below $1.6 \%$ over the entire parameter space of interest $(0.5 \mathrm{GHz} \leq f \leq 20 \mathrm{GHz}$ and $5 \leq$ $\left.\epsilon_{r}^{\prime} \leq 80\right)$. These low errors, which are comparable to the relative errors reported in [24], verify the integrity of the RFM coefficients.

Next, we validated the RFM for solving the inverse problem, i.e., extracting the frequency-dependent complex permittivity of a dispersive lossy dielectric from the aperture-plane reflection coefficient. We applied the RFM to the FDTD-computed aperture-plane reflection coefficient for an idealized probe immersed in a reference liquid. In the Cole-Cole diagrams of Fig. 8, the frequency-dependent complex permittivity obtained from the RFM is compared to the complex permittivity assumed in the FDTD model of the dispersive reference liquid [30]. The errors in the magnitude and phase of the complex permittivity are less than $3.2 \%$ and $1.8^{\circ}$, respectively, over the $0.5-20-\mathrm{GHz}$ frequency range.

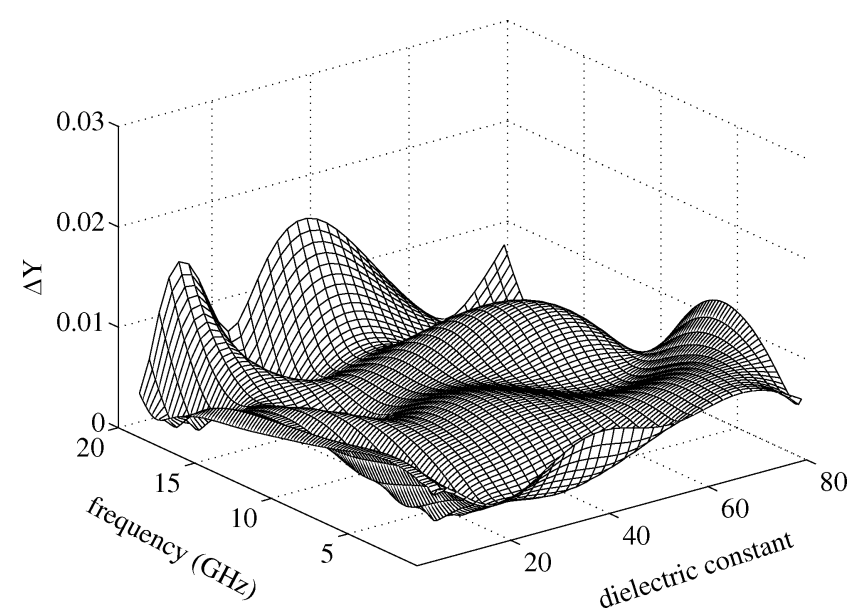

Fig. 7. Magnitude of the relative error between the RFM-predicted and theoretical (FDTD-computed) aperture admittance for lossless dielectrics, as given by (5).

\section{Validation of the Complete Measurement Protocol}

Finally, the performance of the combined data-processing steps (both the deembedding model and RFM) was evaluated using experimental data measured with the UC and UW probes immersed in one of the following reference or test liquids:

1) deionized water;

2) methanol;

3) ethanol;

4) mixture containing equal parts of ethanol and methanol.

The deembedding model was used to process the reflection coefficient measured at the connector/calibration plane and gen- 


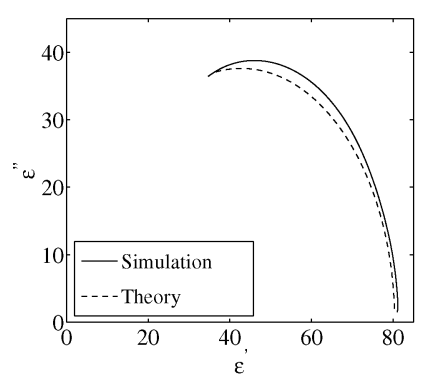

(a)

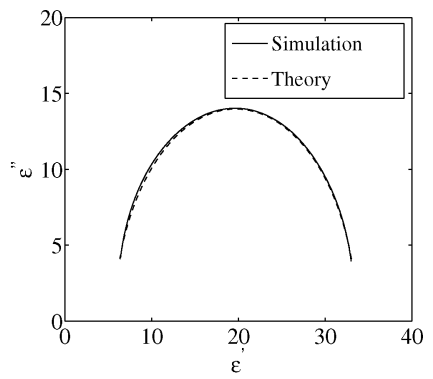

(b)

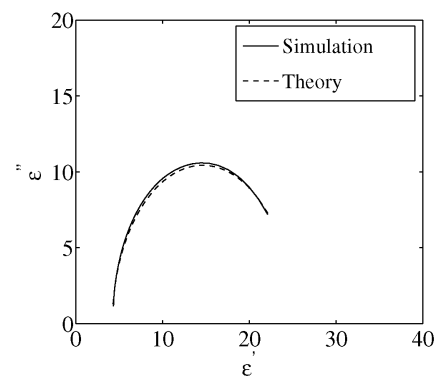

(c)

Fig. 8. Cole-Cole diagrams showing the frequency-dependent complex permittivity extracted from FDTD-computed aperture-plane reflection coefficients using the RFM compared to the theoretical permittivity for three reference liquids. (a) Water. (b) Methanol. (c) Ethanol.

erate the aperture-plane reflection coefficient. The RFM was then used to process the aperture-plane reflection coefficient and extract the complex permittivity of the reference or test liquid. The measured permittivity results for the pure reference liquids were compared with well-established ("theoretical") permittivity values given by a Debye model with parameters interpolated for temperature [30]. The theoretical data for the alcohol mixture was calculated from the volume fraction equations presented in [31].

The Cole-Cole plots in Figs. 9 and 10 show the comparison between the permittivity measured with each precision probe and the theoretical permittivity. The maximum differences in the magnitude and phase of the complex permittivity over the frequency range of interest $(0.5-20 \mathrm{GHz})$ are listed in Table III. The corresponding errors in the real and imaginary parts of the permittivity are well below $10 \%$ for water, methanol, and the ethanol-methanol mixture, but slightly exceed $10 \%$ for ethanol, as expected from the deembedding model results for ethanol.

\section{DISCUSSION}

Deembedding model errors highly depend on the integrity of the reference liquid data used in the model derivation. The errors can be impacted by the specific types of reference liquids chosen, the care with which reference-liquid reflection coefficients are measured, and the accuracy to which the dielectric parameters of the reference liquids are known. The performance of the deembedding model also depends on the degree to which the probe's aperture dimensions are accurately represented in the FDTD model. Here, we discuss several of these important issues.

The permittivities of the reference liquids chosen for the deembedding model derivation should cover the range of permittivities encountered in the actual spectroscopy application. For example, in our application of interest involving

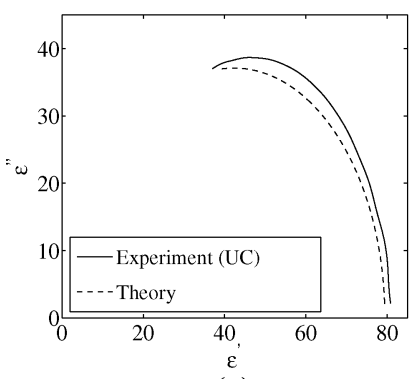

(a)

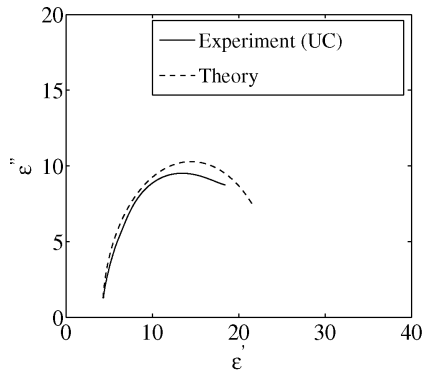

(c)

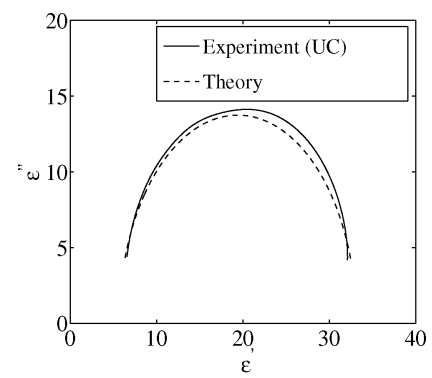

(b)

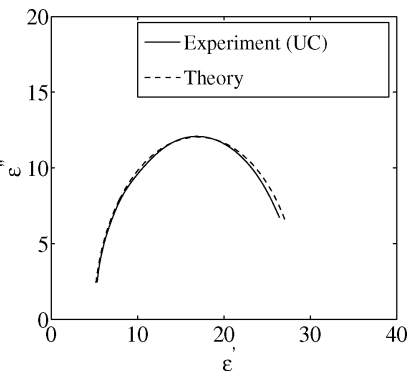

(d)
Fig. 9. Permittivity measured with the primary UC probe versus the theoretical permittivity of: (a) water, (b) methanol, (c) ethanol, and (d) an equal mixture of methanol and ethanol.

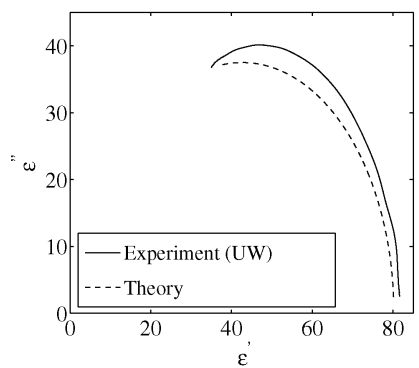

(a)

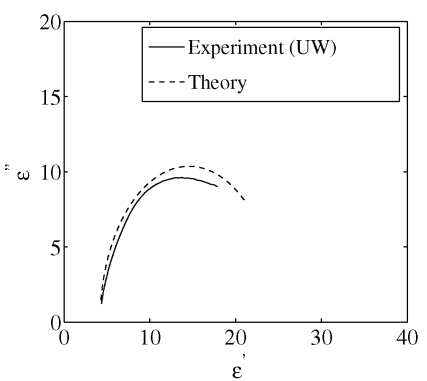

(c)

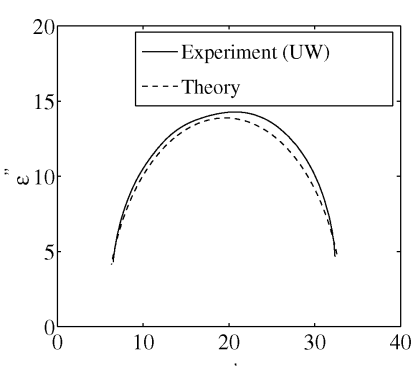

(b)

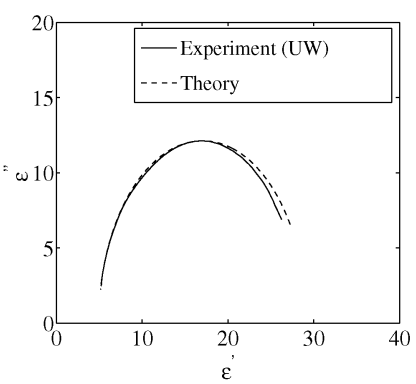

(d)
Fig. 10. Permittivity measured with the primary UW probe versus the theoretical permittivity of: (a) water, (b) methanol, (c) ethanol, and (d) an equal mixture of methanol and ethanol.

breast tissue characterization, the permittivity of the specimens ranges from very low values, as is the case with low-water-content adipose tissue, to very high values, as is the case with high-water-content diseased tissues. This was reflected in the choice of reference liquids in our study. Water, the permittivity of which exceeds that of higher water-content tissues such as fibroglandular tissue and tumors, served as an upper bound on the permittivity range. Ethanol, with its permittivity $\left(\epsilon_{r}=5\right.$ over most of the frequency range of interest) falling below that of infiltrated breast fat, served as a lower bound. Methanol was chosen to fall in the middle of the range of expected 
TABLE III

MAXIMUM PERMITTIVITY ERRORS OF REFERENCE AND TEST LIQUIDS

\begin{tabular}{l|c|c|c|c|c|c|c|c}
\hline & \multicolumn{2}{|c|}{ Water } & \multicolumn{2}{c|}{ Methanol } & \multicolumn{2}{c|}{ Ethanol } & \multicolumn{2}{c}{ Mixture } \\
\hline & Magnitude & Phase & Magnitude & Phase & Magnitude & Phase & Magnitude & Phase \\
\hline UC probe & $4.1 \%$ & $1.5^{\circ}$ & $4.1 \%$ & $1.2^{\circ}$ & $10.6 \%$ & $6.1^{\circ}$ & $4.4 \%$ & $2.0^{\circ}$ \\
\hline UW probe & $4.5 \%$ & $2.3^{\circ}$ & $4.3 \%$ & $1.4^{\circ}$ & $11.4 \%$ & $5.6^{\circ}$ & $3.4 \%$ & $1.9^{\circ}$ \\
\hline
\end{tabular}

permittivities. As discussed in Section IV-A, the errors in the magnitude of the deembedded reflection coefficient were highest for ethanol. We believe this is due to the relatively long relaxation time of ethanol (approximately $140 \mathrm{ps}$ ), which results in a steep rolloff of the theoretical reflection coefficient curves at frequencies below $3 \mathrm{GHz}$. The relaxation times of water and methanol are at least three times smaller (53 and 9 ps, respectively). Fortunately, most biological tissues also exhibit relatively short relaxation time constants [32] for which the deembedding model derived for this study is well suited.

The dielectric properties of reference liquids are temperature dependent, and also change during storage and handling. For example, methanol has very low vapor pressure and starts evaporating almost immediately after it is poured into the measurement beaker. Consequently, the temperature of methanol may decrease several degrees over the course of just a few minutes. To minimize these effects, we strived to take the reference liquid measurements as quickly as possible and we carefully measured temperature at the time the reflection-coefficient measurements were performed.

\section{SUMMARY AND CONCLUSIONS}

A new stainless-steel open-ended coaxial probe was designed, constructed, and evaluated for precision in vivo and ex vivo dielectric spectroscopy of biological tissues, such as breast tissue, over the 0.5-20-GHz frequency range. The use of thermally stable and matched, inert, refractory materials enables hermetic sealing, bio-compatibility, and compatibility with high-temperature sterilization. The design and construction precludes deterioration of the aperture from handling, fluid entrainment, temperature fluctuations, and chemical reaction with biological tissues or fluids. The dimensions were selected to provide optimum sensitivity and small sensing volumes for localized measurements of both lowand high-permittivity tissues.

Robust data-processing techniques were developed and demonstrated for extracting the unknown permittivity of a specimen under test from the reflection coefficient measured with the precision probe and a VNA. The data processing protocol involves the following two steps.

Step 1) A reflection-coefficient deembedding step to translate reflection coefficients measured at the probe's calibration plane to the desired aperture plane.

Step 2) An RFM technique to convert the aperture-plane reflection coefficient to the frequency-dependent, complex permittivity of the specimen.

Validation studies were conducted using reference and test liquids including water, methanol, ethanol, and a methanol-ethanol mixture. The results showed excellent agreement with maximum differences of $\sim 10 \%$ or less between measured permittivities and values derived from published theoretical Debye models.

In conclusion, these probes and data-processing protocols may reliably be used to obtain highly accurate and precise in vivo and ex vivo microwave dielectric properties of biological tissues.

\section{REFERENCES}

[1] C. Beasley, S. Hagness, J. Booske, T. M. Breslin, F. Xu, M. J. Lindstrom, D. Popovic, L. McCartney, M. Okoniewski, W. Temple, A. Magliocco, and T. Ogilvie, "Ex-vivo dielectric characterization of normal, benign, and malignant breast tissue at microwave frequencies: Preliminary results from a multiinstitutional study," in Proc. IEEE AP-S Int. Symp., vol. 4, Columbus, OH, Jun. 2003, p. 39.

[2] S. C. Hagness, A. Taflove, and J. E. Bridges, "Two-dimensional FDTD analysis of a pulsed microwave confocal system for breast cancer detection: Fixed-focus and antenna-array sensors," IEEE Trans. Biomed. Eng., vol. 45, no. 12, pp. 1470-1479, Dec. 1998.

[3] _ , "Three-dimensional FDTD analysis of a pulsed microwave confocal system for breast cancer detection: Design of an antenna-array element," IEEE Trans. Antennas Propag., vol. 47, no. 5, pp. 783-791, May 1999.

[4] P. M. Meaney, M. W. Fanning, D. Li, S. P. Poplack, and K. D. Paulsen, "A clinical prototype for active microwave imaging of the breast," IEEE Trans. Microw. Theory Tech., vol. 48, no. 11, pp. 1841-1853, Nov. 2000.

[5] E. C. Fear and M. A. Stuchly, "Microwave detection of breast cancer," IEEE Trans. Microw. Theory Tech., vol. 48, no. 11, pp. 1854-1863, Nov. 2000.

[6] X. Li and S. C. Hagness, "A confocal microwave imaging algorithm for breast cancer detection," IEEE Microw. Wireless Compon. Lett., vol. 11, no. 3, pp. 130-132, Mar. 2001.

[7] A. E. Bulyshev, S. Y. Semenov, A. E. Souvorov, R. H. Svenson, A. G. Nazarov, Y. E. Sizov, and G. P. Tatsis, "Computational modeling of three-dimensional microwave tomography of breast cancer," IEEE Trans. Biomed. Eng., vol. 48, no. 9, pp. 1053-1056, Sep. 2001.

[8] E. Fear, X. Li, S. Hagness, and M. Stuchly, "Confocal microwave imaging for breast cancer detection: Localization of tumors in three dimensions," IEEE Trans. Biomed. Eng., vol. 49, no. 8, pp. 812-822, Aug. 2002.

[9] E. J. Bond, X. Li, S. C. Hagness, and B. D. Van Veen, "Microwave imaging via space-time beamforming for early detection of breast cancer," IEEE Trans. Antennas Propag., vol. 51, no. 8, pp. 1690-1705, Aug. 2003.

[10] Z. Q. Zhang, Q. Liu, C. Xiao, E. Ward, G. Ybarra, and W. T. Joines, "Microwave breast imaging: 3-D forward scattering simulation," IEEE Trans. Biomed. Eng., vol. 50, no. 10, pp. 1180-1189, Oct. 2003.

[11] E. C. Fear, J. Sill, and M. A. Stuchly, "Experimental feasibility study of confocal microwave imaging for breast tumor detection," IEEE Trans. Microw. Theory Tech., vol. 51, no. 3, pp. 887-892, Mar. 2003.

[12] X. Li, S. K. Davis, S. C. Hagness, D. W. van der Weide, and B. D. Van Veen, "Microwave imaging via space-time beamforming: Experimental investigation of tumor detection in multilayer breast phantoms," IEEE Trans. Microw. Theory Tech., vol. 52, no. 8, pp. 1856-1865, Aug. 2004.

[13] A. J. Fenn, G. L. Wolf, and R. M. Fogle, "An adaptive microwave phase array for targeted heating of tumours in intact breast: Animal study results," Int. J. Hyperthermia, vol. 15, no. 1, pp. 45-61, 1999.

[14] M. Converse, E. J. Bond, S. C. Hagness, and B. D. Van Veen, "Ultrawideband microwave space-time beamforming for hyperthermia treatment of breast cancer: A computational feasibility study," IEEE Trans. Microw. Theory Tech., vol. 52, no. 8, pp. 1876-1889, Aug. 2004.

[15] M. Stuchly and S. Stuchly, "Coaxial line reflection methods for measuring dielectric properties of biological substances at radio and microwave frequencies: A review," IEEE Trans. Instrum. Meas., vol. IM-29, pp. 176-183, Sep. 1980. 
[16] T. Athey, M. Stuchly, and S. Stuchly, "Measurement of radio frequency permittivity of biological tissues with an open-ended coaxial line: Part I," IEEE Trans. Microw. Theory Tech., vol. MTT-30, no. 1, pp. 82-86, Jan. 1982

[17] D. Berube, F. Ghannouchi, and P. Savard, "A comparative study of four open-ended coaxial probe models for permittivity measurements of lossy dielectric/biological materials at microwave frequencies," IEEE Trans. Microw. Theory Tech., vol. 44, no. 10, pp. 1928-1934, Oct. 1996.

[18] D. Popovic and M. Okoniewski, "Effects of mechanical flaws in openended coaxial probes for dielectric spectroscopy," IEEE Microw. Wireless Compon. Lett., vol. 12, no. 10, pp. 401-403, Oct. 2002.

[19] — - "Precision open-ended coaxial probe for dielectric spectroscopy of breast tissue," in Proc. IEEE AP-S Int. Symp., vol. 1, San Antonio, TX, Jun. 2002, pp. 815-818

[20] M. Okoniewski, J. Anderson, E. Okoniewska, K. Caputa, and S. S. Stuchly, "Further analysis of open-ended dielectric sensors," IEEE Trans. Microw. Theory Tech., vol. 43, no. 8, pp. 1986-1989, Aug. 1995.

[21] D. M. Hagl, D. Popovic, S. C. Hagness, J. H. Booske, and M. Okoniewski, "Sensing volume of open-ended coaxial probes for dielectric characterization of breast tissue at microwave frequencies," IEEE Trans. Microw. Theory Tech., vol. 51, no. 4, pp. 1194-1206, Apr. 2003.

[22] D. Misra, M. Chabbra, B. Epstein, M. Mirotznik, and K. Foster, "Noninvasive electrical characterization of materials at microwave frequencies using an open-ended coaxial line: Test of an improved calibration technique," IEEE Trans. Microw. Theory Tech., vol. 38, no. 1, pp. 8-14, Jan. 1990

[23] J. Anderson, C. Sibbald, and S. Stuchly, "Dielectric measurements using a rational function model," IEEE Trans. Microw. Theory Tech., vol. 42, no. 2, pp. 199-204, Feb. 1994.

[24] S. Stuchly, C. Sibbald, and J. Anderson, "A new aperture admittance model for open-ended waveguides," IEEE Trans. Microw. Theory Tech., vol. 42, no. 2, pp. 192-198, Feb. 1994.

[25] A. Taflove and S. Hagness, Computational Electrodynamics: The FiniteDifference Time-Domain Method, 2nd ed. Boston, MA: Artech House, 2000

[26] B. Colpitts, "Temperature sensitivity of coaxial probe complex permittivity measurements: Experimental approach," IEEE Trans. Microw. Theory Tech., vol. 41, no. 2, pp. 229-233, Feb. 1993

[27] D. K. Misra, "Electrical characterization of the materials by a short electric probe," in 8th IEEE Instrumentation Measurement Technology Conf. Rec., Atlanta, GA, May 1991, pp. 502-505.

[28] D. Popovic and M. Okoniewski, "Response characterization of the precision open-ended coaxial probe for dielectric spectroscopy of breast tissue," in Proc. IEEE AP-S Int. Symp., vol. 4, Columbus, OH, Jun. 2003, pp. 54-57.

[29] R. F. Bauer and P. Penfield, "De-embedding and unterminating," IEEE Trans. Microw. Theory Tech., vol. MTT-22, no. 3, pp. 282-288, Mar. 1974

[30] F. Buckley and A. Maryott, "Tables of dielectric dispersion data for pure liquids and dilute solutions," Nat. Bureau Standards, Nat. Bureau Standards Circular 589, 1958

[31] J. Bao, M. Swicord, and C. Davis, "Microwave dielectric characterization of binary mixtures of water, methanol, and ethanol," J. Chem. Phys., vol. 104, no. 12, pp. 4441-4450, Mar. 1996.

[32] C. Gabriel, R. Lau, and S. Gabriel, "The dielectric properties of biological tissues: III. Parametric models for the dielectric spectrum of tissues," Phys. Med. Biol., vol. 41, pp. 2271-2293, 1996.

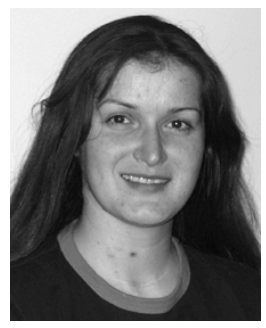

Dijana Popovic (S'00) received the B.S. and M.S. degrees from the University of Calgary, Calgary, AB, Canada, in 2000 and 2002, respectively, and is currently working toward the Ph.D. degree in electrical engineering at the University of Calgary.

Her current research interests are medical applications of microwave technologies, particularly for microwave breast cancer detection.

Ms. Popovic was on the Executive Committees of the IEEE Engineering in Medicine and Biology Society (EMBS) Student Club and the Engineering Graduate Student's Association, University of Calgary. She was the recipient of the 2002 IEEE Microwave Theory and Techniques Society (IEEE MTT-S) Graduate Scholarship, the National Sciences and Engineering Research Council (NSERC) Award, and the Alberta Informatics Circle of Research Excellence (iCORE) Scholarship.

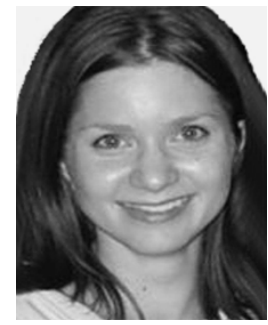

Leah McCartney (S'02) received the B.S. degree in computer engineering from the University of Manitoba, Winnipeg, MB, Canada, in 2002, and is currently working toward the M.S. degree in electrical engineering at the University of Calgary, Calgary, $\mathrm{AB}$, Canada.

Her research interests are the application of microwave technologies to cancer research, as well as other sub-dermal diseases. Her research to date has focused on breast cancer measurements and mathematical modeling.

Ms. McCartney is the acting treasurer of the Electrical and Computer Engineering Graduate Students Association (ECEG), as well as a member of the Graduates Student Association (GSA), University of Calgary. She was the recipient of the National Sciences and Engineering Research Council (NSERC) Award and the Alberta Informatics Circle of Research Excellence (ICORE) Scholarship.

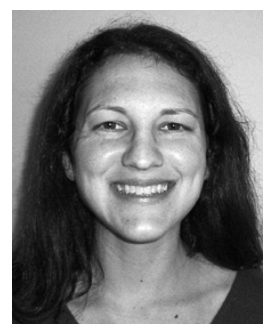

Cynthia Beasley (S'03) received the B.S. degrees in electrical engineering and biological engineering from North Carolina State University, Raleigh, in 2001, and the M.S. degree in electrical engineering from the University of Wisconsin-Madison, in 2003.

Since January of 2004, she has been a Research Engineer with Research Triangle Institute International, Research Triangle Park, NC. She is currently with the Center for Thermoelectrics Research, where her responsibilities include device testing, modeling, and marketing. Her research interests are electrical circuit design, electromagnetics, and biological applications of thermoelectrics.

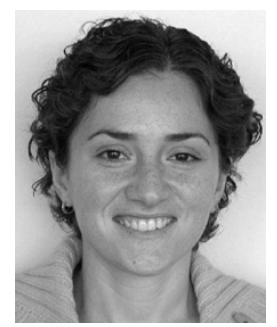

Mariya Lazebnik (S'00) received the B.S. degree in electrical engineering (with highest honors) from the University of Illinois at Urbana-Champaign, in May 2003, and is currently working toward the M.S. degree in electrical and computer engineering at the University of Wisconsin-Madison.

Her research interests include dielectric spectroscopy of biological tissues, microwave imaging, and ultrawide-band antennas.

Ms. Lazebnik is a member of the IEEE Antennas and Propagation Society (IEEE AP-S) and the IEEE Microwave Theory and Techniques Society (IEEE MTT-S). She was the recipient of a 2003-2006 National Science Foundation (NSF) Graduate Research Fellowship and a 2003 IEEE MTT-S Pre-Graduate Scholarship.

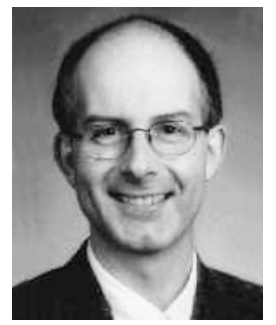

Michal Okoniewski (M'89-SM'97) received the M.Sc. and Ph.D. degrees from the Technical University of Gdansk, Gdansk, Poland, in 1984 and 1990, respectively.

He is currently a Professor with the Department of Electrical and Computer Engineering, University of Calgary, Calgary, AB, Canada, where he holds the Canada Research Chair in Applied Electromagnetics. $\mathrm{He}$ is also an Adjunct Professor with the Department of Electrical and Computer Engineering, University of Victoria, Victoria, BC, Canada. He is also affiliated

with TR-Labs, Canada

His research encompasses computational electrodynamics, novel antenna arrays, such as self-configuring refractarrays, RF-microelectromechanical systems (MEMS), millimeter-wave metamaterials (artificial materials for wave guidance), and photonic circuits. He is also actively involved in interdisciplinary research. Together with his colleagues from both the faculties of Electrical Engineering and Medicine, he is involved with the development of a new breast cancer detection technique and characterization of properties of diseased and normal tissue. With his colleagues from the Department of Electrical and Computer Engineering, he is developing reconfigurable computational hardware capable of order of magnitude faster computations (compared with traditional computers) of complex electromagnetic problems.

Dr. Okoniewski is an associate editor for IEEE TRANSACTIONS ON ANTENNAS AND PROPAGATION. He is a member of Standard Groups. He was the recipient of the 2003 Outstanding Supervision Achievement Award presented by the Faculty of Graduate Studies. 


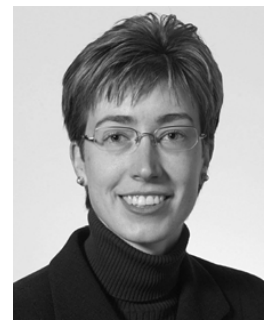

Susan C. Hagness (S'91-M'98-SM'04) received the B.S. (with highest honors) and Ph.D. degrees in electrical engineering from Northwestern University, Evanston, IL, in 1993 and 1998, respectively. While working toward the Ph.D. degree, she was a National Science Foundation (NSF) Graduate Fellow and a Tau Beta Pi Spencer Fellow.

Since August 1998, she has been with the University of Wisconsin-Madison, where she is currently an Associate Professor with the Department of Electrical and Computer Engineering and a faculty affiliate with the Department of Biomedical Engineering. Her research interests encompass FDTD theory and applications in photonics and bioelectromagnetics, as well as theoretical and experimental electromagnetic imaging, sensing, and thermal therapy techniques in biology and medicine. Her research activities include the development of ultra-wideband microwave breast cancer detection and treatment technologies. She coauthored Computational Electrodynamics: The Finite-Difference Time-Domain Method. (Boston, MA: Artech House, 2000, 2nd ed.).

Dr. Hagness is an elected member of the IEEE Antennas and Propagation Society (IEEE AP-S) Administrative Committee (AdCom) and member-at-large of the U.S. National Committee of the International Union of Radio Science (URSI). She is currently an associate editor for the IEEE ANTENNAS AND Wireless PRopagation LetTERS. She was the recipient of the Presidential Early Career Award for Scientists and Engineers presented by the White House in 2000. She was the recipient of the Booker Fellowship Award presented by the United States National Committee of the International Union of Radio Science in 2002. She was also named one of the 100 top young innovators in science and engineering in the world by the Massachusetts Institute of Technology (MIT) Technology Review magazine. In 2003, she was the recipient of the University of Wisconsin Emil Steiger Distinguished Teaching Award. She was also the recipient of the IEEE Engineering in Medicine and Biology Society Early Career Achievement Award in 2004.

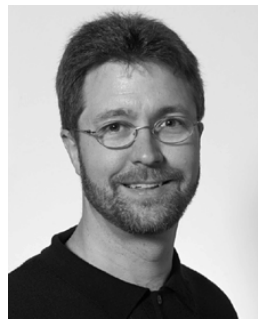

John H. Booske (S'82-M'85-SM'93) received the $\mathrm{Ph} . \mathrm{D}$. degree in nuclear engineering from The University of Michigan at Ann Arbor, in 1985.

From 1985 to 1989, he was a Research Scientist with the University of Maryland at College Park. In 1990 , he joined the faculty of the University of Wisconsin-Madison, where he is currently a Professor of electrical and computer engineering. In 2001, he became the Director of the Materials Science Program, University of Wisconsin-Madison. His research interests include the experimental and theoretical study of coherent electromagnetic radiation sources and their applications with emphasis in the RF, microwave, millimeter-wave, and terahertz regimes. His recent research activities include vacuum electronics, microwave and RF heating of materials, and biological/biomedical applications of microwave and RF fields. He coedited Microwave and Radio Frequency Applications (Westerville, OH: Amer. Ceramic Soc., 2003), and coedited Microwave and Millimeter-Wave Power Electronics (Piscataway, NJ: IEEE Press, 2004).

Professor Booske was a guest editor of the IEEE TRANSACTIONS ON PLASMA SCIENCE. He served as co-director of a U.S. Department of Defense (DoD) MURI99 Consortium on Innovative Microwave Vacuum Electronics and as director of a MURI04 consortium on cathode field emission and RF window breakdown in high-power microwave sources. He was the recipient of the University of Wisconsin Vilas Associate Award for research and the U.S. National Science Foundation (NSF) Presidential Young Investigator Award. He was also the recipient of numerous teaching awards including the University of Wisconsin Chancellor's Distinguished Teaching Award and the Benjamin Smith Reynolds Award for Excellence in Teaching Engineering. 